\title{
Correspondence
}

British Heart fournal, 1977, 39, 1043-1044

\section{Mitral valve prolapse in patients with coronary artery disease}

Sir,

One should differentiate angiographic prolapse from midsystolic click, the auscultatory hallmark of the mitral valve prolapse-click syndrome. In my articles quoted by the authors, I have refuted the causal association between coronary artery disease and the midsystolic click rather than between coronary artery disease and angiographic mitral valve prolapse. In coronary artery disease, angiographic-auscultatory dissociation commonly occurs, mitral valve prolapse resulting from papillary muscle dysfunction being shown by angiography in the absence of a click. Furthermore, angiographicechocardiographic dissociation may occur in coronary artery disease with the echocardiogram showing absence of mitral valve prolapse despite the obvious angiographic demonstration of prolapse. This dissociation is not evident in the idiopathic click syndrome in which echocardiography is positive in about 80 to 90 per cent of patients. It seems that a myxomatous redundant mitral valve is a prerequisite for the occurrence of a click and of an echocardiogram diagnostic of prolapse. Left ventricular angiography seems to be less specific, showing prolapse not only in the idiopathic click syndrome but in papillary muscle dysfunction, and in hypertrophic subaortic stenosis, etc. One should not, therefore, equate angiographic prolapse with the idiopathic mitral valve prolapse-click syndrome. Unfortunately, the present authors (Raizada et al., 1977) have not reported the auscultatory findings in their patients. I assume that none of them had a midsystolic click. I disagree with the authors' interpretation of their Fig. 1 and 2. Fig. 1 does not show prolapse of the posteromedial scallop of the posterior mitral leaflet. The area indicated by the arrow corresponds to a segment of the inferior wall of the ventricle adjacent to the indentation made by the posterior papillary muscle and is quite distant from the mitral valve annulus which is located near the tip of the catheter.

The echocardiogram on Fig. 2 is not diagnostic of mitral valve prolapse. There is a minimal nonspecific sagging of the anterior leaflet and the posterior leaflet is flat but shows no pansystolic or late systolic sagging.
It would be interesting to know the history of the patient shown in Fig. 3. My guess is that this patient who has obvious angiographic and echocardiographic prolapse may have two separate diseases, namely, coronary artery disease and myxomatous degeneration of the mitral valve.

Robert M. Jeresaty
Saint Francis Hospital
114 Woodland Street
Hartford, Connecticut, 06105, USA

\section{Reference}

Raizada, V., Benchimol, A., Desser, K. B., Reich, F. D., Sheasby, Connie, and Graves, Carol (1977). Mitral valve prolapse in patients with coronary artery disease. Echocardiographic-angiographic correlation. British Heart fournal, 39, 53-60.

This letter was shown to the authors who reply as follows.

Fig. 1 does indeed show prolapse of the posteromedial scallop of the posterior mitral leaflet. In our experience, prolapse of the posterior wall of the left ventricle toward the left atrium is distinctly unusual. Here, the most inferior scallop is prominently displayed.

If Dr. Jeresaty does not consider the echocardiogram presented in Fig. 2 diagnostic of mitral valve prolapse, then the reasons for his 'dissociation' are apparent. The prodigious posterior leaflet sagging observed on this tracing is clear.

Current statistics relating to the incidence of nonejection systolic clicks and echographic evidence of prolapse in the general population suggest that the vast majority of patients with the 'click-prolapse' syndrome have no history compatible with cardiac disease. Given these considerations, it is no revelation to learn that Fig. 3 represents angiograms and ultrasonic studies from a subject with a history of angina pectoris secondary to coronary artery disease.

Only a single patient in our study group had an audible midsystolic click. If Dr. Jeresaty considers 
the click a requisite feature of idiopathic mitral valve prolapse, then he is in effect placing thousands of subjects with isolated apical systolic murmurs and definitive echocardiographic evidence of prolapse in yet another category which does not accord with his somewhat confusing and ever changing definition of the 'syndrome'.
Kenneth B. Desser and Alberto Benchimol

Good Samaritan Hospital

Institute for Cardiovascular Diseases, 1033 East McDowell Road, PO Box 2989

Phoenix, Arizona, USA. 